\title{
Models of Pseudolinear Interface Electrostatics for Air-Semiconductor Media
}

\author{
Toshko Boev ${ }^{1}$, Boryan Radoev ${ }^{2}$, Veliko Donchev ${ }^{1}$ \\ ${ }^{1}$ Faculty of Mathematics and Informatics University of Sofia, 5, J. Boucher Ave., Sofia, Bulgaria \\ ${ }^{2}$ Faculty of Chemistry University of Sofia, 3, J. Boucher Ave., Sofia, Bulgaria \\ boev@fmi.uni-sofia.bg,radoev@chem.uni-sofia.bg, velikod@gmail.com
}

\begin{abstract}
Via an idealizing approach, due to Gibbs, it is discussed a mathematical model of surface electrostatics in case of a technologically manipulated interface. The surface distribution of the electric potential is effectively found to be examined the role of functional nano-roughnesses.

Index Terms - heterogeneous systems, transmission problems.
\end{abstract}

\section{Introduction}

We propose in the present study a model covering actually a large class of heterogeneous systems. The model is applied here to electrostatics of air-crystal (or gas-crystal) material systems, which contain a separating semiconductor surface (interface). The separating folio is produced by technological annealing, resulting in functional step nano-roughnesses. As known, the importance of the surface roughnesses, consequently - of the interface electrostatics, is motivated from actual technological questions (e.g. [5], [7]), corresponding to the contemporary growing usage of various electronic devices. The governing technological role of the interface (for the whole compound system, of given electronic device), especially that of the relevant nano-roughness, yields the effective determination of the interface electric potential as a key problem. Note additionally the necessity to examine electrostatic interactions of the roughness segments and the surface charge density, via distributions nonlinearly depending on the electric potential. For solving the main problems we introduce below an idealized, according to Gibbs ([6]), heterogeneous system. The Gibbs idealizing approach treats the thin transitional layers as interfaces, under a principle called the excesses one ([6], see also [10]). Thus, taking initially a 3D air-crystal material system, with a thin midmost transition layer, of a semiconductor matter, we come to the following Gibbs type of idealization. The full system is supposed to consist in two bulk phases - of upper (air or gas) and lower (crystal) media, and a flat semiconducting interface, with a distribution of fine technological surface roughnesses, shaped as a straight line of defects (as a compatible illustration, see Fig. 1, [4]). Such interface structure is essentially supported by real effects, experimentally established under annealing of $\operatorname{InP}(110)$ samples at temperatures up to $480 \mathrm{~K}$, followed by heat normalizing ([5], [7]). More over, a credible visual result of [5] and [7] (see Fig. 2, [7]), established by scanning tunneling microscopy of the surface crystal layer, completely confirms the modelconstruction derived from the Gibbs theory. Next, having settled the Gibbs framework, we have introduced a typical 3-21 D heterogeneous material system, $G^{*} \equiv\left\{B^{-} \cup S \cup B^{+}\right\}$, with $S=S^{-} \cup l \cup S^{+}$. Certainly, the system incorporates variety of homogeneous material components, of three different dimensions: $B^{+}$and $B^{-}$enter as homogeneous bulk phases, but of quite differing electrostatics (they are respectively of air, considered here as vacuum, and crystal); $S$ is the interface, which enters as an autonomous 2D semiconductor folio separating the bulk phases and consisting in two electrostatic equivalent and homogeneous halves, $S^{-}$, $S^{+}$. And the roughnesses composition enters in the idealized system $G^{*}$ as a detached material contour $l$ - a straight line, splitting $S$ into the surface phases $S^{-}, S^{+}$. Contour $l$ plays the role of an intrinsic 1D phase (of electrostatic autonomous and homogeneous matter). For a technically convenient presentation of system $G^{*}$, let us introduce a Cartesian $(x, y, z)$ - coordinate system, with line $l$ as the $O y$ axis and surface $z=0$ as the interface of $G^{*}$. The vacuum and crystal bulk phases $\left(B^{+}\right.$and $B^{-}$) fill the upper and lower semispaces, $z>0$ and $z<0$, respectively, and for the surface phases we have: $S^{-}(x<0, z=0)$ and $S^{+}(x>0, z=0)$. As the electric potential $u=u(x, y, z)$ is the key quantity determining electrostatics, our model goals to express it for the whole vacuum-surface-crystal heterogeneous system. Due to the key role of the technological roughnesses, it is of special interest to find first of all the surface potential distribution $u(x, y, z=0)$ and, secondly, its impact on the bulk distributions $u(x, y, z \neq 0)$ in the vacuum and crystal phases. The said point of view is the motivation to accent here on finding effective expressions of the interface potential.

Note now the homogeneity of heterogeneous system $G^{*}$ on the $y$-direction, because of the assumed homogeneity of the $1 \mathrm{D}$ phase $l$. Therefore both, the electric potential

\footnotetext{
* This work is partially supported by Grant No 026/2012 of the Sofia University Science Foundation and Grant No DDVU 02/90 of the Bulgarian National Science Foundation.
} 
$u=u(x, y, z)$ and vector $\mathbf{D}=\mathbf{D}(x, y, z)$, of the electric induction ([9]), will actually depend on $x, z$, i.e. $u=u(x, z)$ and $\mathbf{D}=\mathbf{D}(x, z)$. (Vector $\mathbf{D}$ is also known as electric displacement, e.g. [8].) Here we propose interface electrostatics with a charge density nonlinearly modeled, regarding a specific modulation $u_{K}(x, 0)$ of potential $u(x, 0)$. The modulated potential $u_{K}(x, 0)$ enables a linear treatment of the problem for determining the surface potential. Thus a kind of pseudolinear electrostatics is proposed and studied. Concerning $u_{K}$ modulation, let us shortly accent on the assumed structure of the surface charge density. We deal below with a quadratic type of dependence on $u_{K}$, expressed by the weighted $x$-mean value $\mu_{K}[u]$, as follows:

$$
\begin{aligned}
& \text { (0.1) } \mu_{K}[u]=\mu_{K}[u](z) \equiv \frac{K}{2} \int_{-\infty}^{\infty} u(x, z) d x \\
& \text { (0.2) } u_{K}=u_{K}(x, z) \equiv \zeta_{K}(x) \mu_{K}[u](z)
\end{aligned}
$$

$\left(\zeta_{K}(x) \equiv \exp (-K|x|) ; K\right.$ is a positive parameter.

The usage of potential $u_{K}$ in the nonlinear relations, instead of $u$, simplifies the technical procedures saving well enough the essence of the exact potential distribution. This is essentially supported by the asymptotic equivalence:

$(u)_{L} /\left(\zeta_{K}\right)_{L} \sim \int_{-\infty}^{+\infty} u(x, z) d x / \int_{-\infty}^{+\infty} \zeta_{K}(x) d x=\frac{K}{2} \int_{-\infty}^{\infty} u(x, z) d x$

$(L>>1)$,

where $\quad(u)_{L}=(u)_{L}(z) \equiv(1 / 2 L) \cdot \int_{-L}^{L} u(x, z) d x \quad, \quad$ by analogy - for $\left(\zeta_{K}\right)_{L}$. Note that potential $u_{K}$ has a compatible impact with that of $u$ because of the identical averages:

$\mu_{K}\left[u_{K}\right]=\mu_{K}[u]$.

Using the quadratic expression $\varepsilon_{0}\left(-\varepsilon_{s} k_{s}^{2} u+\varepsilon_{s}^{2} k_{s}^{4} u^{2} / 2\right)$, with $u^{2}$ replaced by $u_{K}^{2}$, for the interface charges, we introduce the following relations as the basic form of our model (a derivation sketch is given in Sect. 2 , below):

$$
\begin{aligned}
& D_{+}^{z}(x, 0)-D_{-}^{z}(x, 0)+\nabla_{s} . \mathbf{D}_{s}=\varepsilon_{0}\left(-\varepsilon_{s} k_{s}^{2} u+\varepsilon_{s}^{2} k_{s}^{4} u_{K}^{2} / 2\right) \\
& (z=0, x \neq 0) \\
& \left(\mathbf{D}_{s}\right)^{x,+}-\left(\mathbf{D}_{s}\right)^{x,-}=\rho_{l} .
\end{aligned}
$$

Above $\nabla_{s}$ is the nabla operator, tangential (to $z=0$ ); $D_{+}^{z}(x, 0)=\lim _{z \rightarrow+0} D^{z}(x, z)$, by analogy - for $D_{-}^{z}(x, 0)$, where $D^{z}$ is the normal to $z=0$ component of vector $\mathbf{D}$, and the limits are supposed finite, $\forall x ; \mathbf{D}_{s} \equiv \mathbf{D}(x .0)$ and $\nabla_{s} . \mathbf{D}_{s}$ is the formal scalar product of vectors (nabla and $\left.\mathbf{D}_{s}\right)$, i.e. $\nabla_{s} \cdot \mathbf{D}_{s}=\operatorname{div} \mathbf{D}_{s} ;\left(\mathbf{D}_{s}\right)^{x,-},\left(\mathbf{D}_{s}\right)^{x,+}$ are the relevant limits (also assumed finite), at $x \neq 0$, for the normal to $l$ (and coplanar to $z=0$ ) component $\left(\mathbf{D}_{s}\right)^{l}$ of $\mathbf{D}_{s}$. Parameters $k_{s}, \varepsilon_{s}$ are the main electrostatic characteristics to the surface-matter; here they enter as conventional positive constants. The material sense of $k_{s}$ is present by the quantity $k_{s}^{-1}=1 / k_{s}$, called surface screening length (by analogy to the bulk one, $k_{b}^{-1}=1 / k_{b}$, known from the space electrostatics e.g. [10]). Parameter $\varepsilon_{s}$ is the surface dielectric permittivity (by analogy to the bulk' one, $\varepsilon_{b}$ ). Condition (1.2) is based on the key line (point) characteristic $\beta_{l}$, where $\beta_{l}=\rho_{l} / \varepsilon_{0}$, with $\rho_{l}$ - the electric charge density upon the line (point) phase; $\varepsilon_{0}=8.85 \mathrm{pF} / \mathrm{m}$ is the known absolute dielectric permitivity.

The model yields the basic mathematical problem for existence of solutions $\left(\mathbf{D}_{s}, u\right)$ to system (1.1), (1.2). For the analysis, a surface transmission problem is solved, in Sect. 2, regarding potential $u(x, 0)$. It is done by reducing to a nonlinear boundary integral equation, which admits effective resolving. Finding then an explicit expression for the surface potential, we estimate, in concluding remarks (Sect. 3), the effects of the nonlinear surface distribution of charges.

\section{Elements of modelling, surface transmission problems and integral equations.}

The key tool for description of electrostatic phenomena in complex media relates to the Maxwell system (in case of dielectrics, e.g. [9]):

$$
\text { a) } \quad \nabla . \mathbf{D}=\rho \text {; b) } \mathbf{D}=-\varepsilon_{0} \varepsilon \nabla u \text {. }
$$

Above $\rho$ is the charge density; $\varepsilon$ is the relative dielectric permitivity for the relevant part of the medium $\left(\varepsilon=\varepsilon_{b}^{-}\right.$, at $z<0 ; \varepsilon=\varepsilon_{b}^{+}$, at $z>0$, with $\varepsilon_{b}^{+}=1$, from the vacuum assumption; $\varepsilon=\varepsilon_{s}$, at $z=0, x \neq 0$ ). Equations (2.1) hold for the total (3D) system and, as known, potential $u$ is a continuous function of $(x, y, z)$, in spite of the various material phases. Because of the presumed complex heterogeneity of $G^{*}$, we shall apply a two-leveled scheme of singular decompositions to $\mathbf{D}$ and $\rho$, in reworking of the Maxwell system. Thus we express the electrostatic field 
$(\mathbf{D}, u)$ of system $G^{*}$ by decompositions in two levels (bulk and surface), of the following type:

a) $\rho=\rho_{b}^{-} \eta^{-}+\rho_{b}^{+} \eta^{+}+\rho_{s} \delta(z)$;

b) $\rho_{s}=\rho_{s}^{-} \eta_{s}^{-}+\rho_{s}^{+} \eta_{s}^{+}+\rho_{l} \delta_{l}$;

$$
\begin{aligned}
& \mathbf{D}=\left(\mathbf{D}_{b}\right)^{-} \eta^{-}(z)+\left(\mathbf{D}_{b}\right)^{+} \eta^{+}(z)+\mathbf{D}_{s} \delta(z) ; \\
& \mathbf{D}_{s}=\left(\mathbf{D}_{s}\right)^{-} \eta_{s}^{-}+\left(\mathbf{D}_{s}\right)^{+} \eta_{s}^{+}+\mathbf{D}_{l} \delta_{l} .
\end{aligned}
$$

Above $\eta^{+}(z) / \eta^{-}(z)$ are respectively the Heaviside forward/backward functions (i.e. $\eta^{+}(z)=1$, at $z>0$, $\eta^{+}(z)=0$, at $\left.z<0, \eta^{-}(z) \equiv \eta^{+}(-z)\right)$ and $\delta(z)$ is the Dirac delta-function, supported at $z=0 ; \eta_{s}^{-}=1$, at $z=0, x<0$ and $\eta_{s}^{-}=0$, at $z=0, x>0$, by analogy: $\eta_{s}^{+}=1$, at $z=0, x>0$ and $\eta_{s}^{+}=0$, at $z=0, x<0$; next, $\quad \delta_{l}$ is delta-function, supported on the line $l: x=0(z=0)$, and we shall also use the notation $\delta(x)$, for $\delta_{l}$. Relations (2.2) - (2.4) present an essential generalization, in two levels (already introduced in [10]), of the Bedeoux-Vlieger ([3]) step formalism. Vector $\mathbf{D}_{s}$ is a function of $x$, assumed in the form of (2.4), with vector components $\left(\mathbf{D}_{s}\right)^{-},\left(\mathbf{D}_{s}\right)^{+}$, having finite and different limit values at $x \rightarrow 0$. Analogous presumptions hold to scalar functions $\rho_{s}^{ \pm}=\rho_{s}^{ \pm}(x)$ (considered as at least continuous respectively at $x \leq 0$ and $x \geq 0) ; \rho_{l}$, presented in (2.2.b), is a scalar and $\mathbf{D}_{l}$ (see (2.4)) is a vector. In (2.2) we shall assume in general nonlinear dependences of the bulk and surfaces charge densities ( $\rho$ and $\rho_{s}$ ) on the electric potential, i.e. $\rho=\rho[u](x, z), \rho_{s}=\rho_{s}[u](x)$. For a motivation we use certain arguments from a known Fermi-Dirac distribution (e.g. [2]) and analogies with the Boltzmann one, via the GouyChapmann theory (e.g. [8]). Substituting now from (2.2) - (2.4) into electrostatic equations (2.1), we get, for the bulk phases:

(2.5.a) $\nabla .\left(\mathbf{D}_{b}\right)^{+}=0(z>0), \nabla .\left(\mathbf{D}_{b}\right)^{-}=\rho_{b}^{-}[u](z<0)$;

$$
\begin{aligned}
& \left(\mathbf{D}_{b}\right)^{+}=-\varepsilon_{0} \varepsilon_{b}^{+} \cdot \nabla u(z>0), \\
& \left(\mathbf{D}_{b}\right)^{-}=-\varepsilon_{0} \varepsilon_{b}^{-} \cdot \nabla u(z<0) .
\end{aligned}
$$

And for the interface we find:

$$
\begin{aligned}
& D_{+}^{z}(x, 0)-D_{-}^{z}(x, 0)+\nabla_{s} \cdot \mathbf{D}_{s}=\rho_{s}[u] \\
& (z=0, x \neq 0) ;
\end{aligned}
$$

$$
\left(\mathbf{D}_{s}\right)^{x,+}-\left(\mathbf{D}_{s}\right)^{x,-}=\rho_{l},
$$

where, for vector $\mathbf{D}_{s}$ it holds

$$
\mathbf{D}_{s}=-\varepsilon_{0} \varepsilon_{s} \cdot \nabla_{s} u(z=0, x \neq 0) .
$$

Calculating the results of above substituting, let us note that the normal to $z=0$ component $D_{s}^{z}$ (of vector $\mathbf{D}_{s}$ ) vanishes $\left(D_{s}^{z}=0\right.$ ), i.e. $\mathbf{D}_{s}$ presents a flat (planar) vector field (see e.g. [10] for details).

Concerning the charge density terms $\rho_{b}^{-}[u], \rho_{s}[u]$, in (2.5.a), (2.6), for the vacuum-surface-crystal system $G^{*}$ we have to forecast a relation in the form of $\rho=\rho_{b}^{-}[u]$ (for the crystal phase), because of the mentioned Fermi-Dirac and Boltzmann distributions. Presuming a total electro-neutrality of the considered material system, we shall take into account first of all general charge distribution laws in the following form, respectively for the crystal bulk and surface phases:

$$
\begin{aligned}
& \rho=\varepsilon_{0}\left[\exp \left(-\varepsilon k^{2} u\right)-1\right], \\
& \rho_{s}=\varepsilon_{0}\left[\exp \left(-\varepsilon_{s} k_{s}^{2} u\right)-1\right] .
\end{aligned}
$$

The potential-magnitudes can be however assumed relatively small, and we can deal with truncations of the infinite sums (presenting $\rho[u]$ and $\rho_{s}[u]$ from (2.9)), i.e. $-\varepsilon k^{2} u+\varepsilon_{s}^{2} k^{4} u^{2} / 2 !-\varepsilon_{s}^{3} k^{6} u^{3} / 3 !+\cdots \quad$ and $-\varepsilon_{s} k_{s}^{2} u+\varepsilon_{s}^{2} k_{s}^{4} u^{2} / 2 !-\varepsilon_{s}^{3} k_{s}^{6} u^{3} / 3 !+\cdots$, possibly closer to the linear terms. Thus we chose linear approximation, $\rho_{1}[u]=-\varepsilon_{0} \varepsilon k^{2} u$, for $\rho[u]$ but quadratic one, $\rho_{s, 2}[u]=\varepsilon_{0}\left(-\varepsilon_{s} k_{s}^{2} u+\varepsilon_{s}^{2} k_{s}^{4} u^{2} / 2\right.$ !), for $\rho_{s}[u]$, because of certain dominating role of the interface. Additionally, in the quadratic term $\varepsilon_{s}^{2} k_{s}^{4} u^{2} / 2$ ! of $\rho_{s, 2}[u]$ we change the factor $u^{2}$ into the similar one of $u_{K}^{2}$ (recall the comments in Sect. 1). Thus we shall deal with the modified density of surface charges, as follows:

$$
\rho_{2}^{K, s}[u]=\varepsilon_{0}\left(-\varepsilon_{s} k_{s}^{2} u+\varepsilon_{s}^{2} k_{s}^{4} u_{K}^{2} / 2\right) .
$$

On the linear (1D) phase, the contour $l=O y$, we assume $\rho_{l}=\varepsilon_{0} \beta_{l}$, with $\beta_{l}-$ given constant. Substituting now $\rho_{s}[u]$ with $\rho_{2}^{K, s}[u]$ in (2.6), from (2.6), (2.7) we find the pseudolinear model (1.1), (1.2) of interface electrostatics for system $G^{*}$. 
For the analysis of problem (1.1), (1.2) we shall rework the terms $D_{+}^{z}(x, 0)-D_{-}^{z}(x, 0)$ and $\left(\mathbf{D}_{s}\right)^{x,+}-\left(\mathbf{D}_{s}\right)^{x,-}$ : from (2.5.b) and (2.8) it follows respectively that

$$
D_{ \pm}^{z}(x, 0)=-\varepsilon_{0} \varepsilon_{b}^{ \pm} u_{z}(x, \pm 0)
$$

and $\left(\mathbf{D}_{s}\right)^{x, \pm}=-\varepsilon_{0} \varepsilon_{s} u_{x}( \pm 0,0)$. Thus the mentioned differences yield respectively the following jump type of terms:

$\Delta\left[\varepsilon_{b} u_{z}\right]=\Delta\left[\varepsilon_{b} u_{z}\right](x, 0)$,

With

$\Delta\left[\varepsilon_{b} u_{z}\right](x, 0)=\varepsilon_{b}^{+} u_{z}(x,+0)-\varepsilon_{b}^{-} u_{z}(x,-0) \quad\left(\right.$ at $\left.\varepsilon_{b}^{+}=1\right)$, and $\varepsilon_{s}\left[u_{x}(+0,0)-u_{x}(-0,0)\right]$. Then problem (1.1), (1.2) takes the form:

$$
\begin{aligned}
& \Delta\left[\varepsilon_{b} u_{z}\right]+\varepsilon_{s} u_{x x}=\varepsilon_{s} k_{s}^{2} u-\varepsilon_{s}^{2} k_{s}^{4} u_{K}^{2} / 2, \\
& z=0, x \neq 0 \\
& \varepsilon_{s}\left[u_{x}(+0,0)-u_{x}(-0,0)\right]=-\beta_{l} .
\end{aligned}
$$

Above $u_{x}, u_{z}, u_{x x}$ are first or second order derivatives regarding the relevant variable and $u_{x}( \pm 0,0)=\lim _{x \rightarrow 0} u_{x}(x, 0)$, respectively at $x>0, x<0$. For next reworking denote by $\varphi(x)$ the surface values $u(x, 0)$ and use that (2.5) yield the Laplace and Helmholtz equations $\nabla^{2} u=0(z>0)$ and $\nabla^{2} u=k_{b}^{2} u(z<0)$, for the space potential $u(x, z) \quad$ (under boundary condition $u(x, 0)=\varphi(x))$. Now we can express, by the $\mathrm{x}$ - Fourier transforms of $u(x, z)$ and $\varphi(x)$, the jump term $\Delta\left[\varepsilon_{b} u_{z}\right]$ as $\Delta\left[\varepsilon_{b} u_{z}\right](\varphi)=L^{0}[\varphi]$. Here $L^{0}: \varphi \rightarrow L^{0}[\varphi]$ is the linear operator, such that $\mathbf{F}\left(L^{0}[\varphi]\right)(\xi)=-\lambda(\xi) \hat{\varphi}(\xi)$, for the Fourier transform $\mathbf{F}\left(L^{0}[\varphi]\right)$ of function $L^{0}[\varphi](x)$, where $\lambda(\xi)=|\xi|+\varepsilon_{b} \sqrt{\xi^{2}+k_{b}^{2}}, \varepsilon_{b}=\varepsilon_{b}^{-}, k_{b}=k_{b}^{-}$. This way we present (1.1), (1.2) as the following problem, for the surface potential $\varphi(x)$.

$$
\begin{aligned}
& L^{0}[\varphi]+\varepsilon_{s} \varphi^{\prime \prime}=\varepsilon_{s} k_{s}^{2} \varphi-\varepsilon_{s}^{2} k_{s}^{4} \varphi_{K}^{2} / 2, \\
& z=0, x \neq 0 \\
& \varepsilon_{s}\left[\varphi^{\prime}(+0)-\varphi^{\prime}(-0)\right]=-\beta_{l}, z=0, x=0 .
\end{aligned}
$$

Above $\varphi_{K}=\varphi_{K}(x)=\mu_{K}[\varphi] \cdot \zeta_{K}(x)$ is the K-modulation of potential $\varphi$ and $\varphi^{\prime}, \varphi^{\prime \prime}$ are respectively the first and second order derivative of function $\varphi$.
Now the essential step consists in solving problem (2.13), (2.14). Given a solution $\varphi(x)$, let us consider the auxiliary equation (directly suggested by relation (2.13)):

$$
w^{\prime \prime}-k_{s}^{2} w+F_{K}[\varphi](x)=0
$$

$\left(F_{K}[\varphi] \equiv L^{0}[\varphi] / \varepsilon_{s}+\varepsilon_{s} k_{s}^{4} \varphi_{K}^{2} / 2\right), x \neq 0$.

Because function $w=\varphi(x)$ is a solution of (2.15), by the argument of the general solution formula, we get the expression:

$$
\varphi=c^{*} \exp \left(-k_{s}|x|\right)+\exp \left(-k_{s}|\cdot|\right) * F_{K}[\varphi] / 2 k_{s} .
$$

Here $F_{1} * F_{2}$ is the known convolution of two (generalized) functions (e.g. [1]) and $c^{*}$ is a constant depending on $\varphi$. Substituting by (2.16) in condition (2.14), constant $c^{*}$ can be determined as $c^{*}=\beta_{l} / 2 \varepsilon_{s} k_{s}$ and the starting form of the needed integral equation is found:

$$
\varphi=\frac{\beta_{l}}{2 \varepsilon_{s} k_{s}} \exp \left(-k_{s}|x|\right)+\exp \left(-k_{s}|\cdot|\right) *\left(\frac{L^{0}[\varphi]}{2 \varepsilon_{s} k_{s}}+\frac{\varepsilon_{s} k_{s}^{3} \varphi_{K}^{2}}{4}\right)
$$

Reworking (2.17) and using the auxiliary function $\phi^{s}(x)$, with

$$
\phi^{s}(x)=\frac{1}{\pi} \int_{0}^{\infty}\left[\lambda(\xi)+\varepsilon_{s}\left(k_{s}^{2}+\xi^{2}\right)\right]^{-1} \cos (x \xi) d \xi,
$$

we establish the following compact expression for the integral equation:

$$
\varphi=\beta_{l} \phi^{s}+\varepsilon_{s}^{2} k_{s}^{4} \phi^{s} * \varphi_{K}^{2} / 2
$$

\section{Concluding remarks.}

Beginning with the basic effective formula for the interface potential, we will accent here on the next three conclusions.

(i) It holds the following explicit formula for the surface potential $\varphi$ :

$$
\varphi(x)=\beta_{l} \phi^{s}(x)+\beta_{l} \Phi_{s}^{b, l}(x),
$$

where

$$
\Phi_{s}^{b, l}(x) \equiv \frac{1}{\pi} \int_{0}^{\infty} \frac{\cos (x \xi) d \xi}{\left[\lambda(\xi)+\varepsilon_{s}\left(k_{s}^{2}+\xi^{2}\right)\right]\left[1+\left(\beta_{l} / 4 k_{b, s}^{2}\right)^{2} \xi^{2}\right]}
$$

To comment this assertion let us integrate equality (2.18) (for a given solution $\varphi(x))$ : we find the simple relation 
$K \varepsilon_{s}^{2} k_{s}^{4} I_{\varphi}^{2} / 8-I_{s}^{-1} I_{\varphi}+\beta_{l}=0$, with $I_{\varphi}=\int_{-\infty}^{\infty} \varphi(x) d x$,

$I_{s}=\int_{-\infty}^{\infty} \phi^{s}(x) d x$, and use that $I_{s}^{-1}=\varepsilon_{b} k_{b}+\varepsilon_{s} k_{s}^{2}$. Then we chose $K=K_{s}^{b, l}$, where $K_{s}^{b, l} \equiv 2 k_{b, s}^{2} \beta_{l}^{-1}$, $k_{b, s}=1+\varepsilon_{b} k_{b} / \varepsilon_{s} k_{s}^{2}$. At $K=K_{s}^{b, l}$ the above equation (regarding $I_{\varphi}$ ) yields the following uniquely determined value: $I_{\varphi}=2 \beta_{l} /\left(\varepsilon_{b} k_{b}+\varepsilon_{s} k_{s}^{2}\right)$. And, for the modulated potential $\varphi_{K}$ (by relation $\varphi_{K}(x)=\mu_{K}[\varphi] \cdot \zeta_{K}(x)$ ), we then find:

$$
\varphi_{K}(x)=2 \varepsilon_{s}^{-1} k_{s}^{-2} k_{b, s} \zeta_{K}(x)\left(K=K_{s}^{b, l}\right) .
$$

To prepare the substitution from (3.2) into (2.18), note firstly that $\zeta_{K}^{2}=\zeta_{2 K}$ and denote afterwards $\psi_{s}^{b, l}(x) \equiv \zeta_{2 K}(x) \quad\left(\right.$ at $\left.\quad K=K_{s}^{b, l} \quad\right) ; \quad$ i.e. $\psi_{s}^{b, l}(x)=\exp \left(-2 K_{s}^{b, l}|x|\right)$. Now from (2.18) and (3.2) we get: $\varphi=\beta_{l} \phi^{s}+2 k_{b, s}^{2} \phi^{s} * \psi_{s}^{b, l}$. By reworking the convolution $\phi^{s} * \psi_{s}^{b, l}$, the result can be conveniently expressed via function $\Phi_{s}^{b, l}$ in the form of (3.1).

(ii) The impact of the modulated surface potential is comparable with that of the leading term $\beta_{l} \phi^{s}(x)$, at low charged anomaly contour.

For a comment of the formulated effect, let us firstly note that the term $\beta_{l} \phi^{s}(x)$ in (3.1) presents the surface potential distribution via the linear theory - when relation (1.1) of the basic model does not includes the modulating factor $u_{K}^{2}$. Thus the term $\beta_{l} \Phi_{s}^{b, l}(x)$ should be considered as a perturbation produced by the squared modulation $u_{K}^{2}$. A comparison of potentials $\phi^{s}(x)$ and $\Phi_{s}^{b, l}(x)$ follows, from the above given integral formulas (for $\phi^{s}, \Phi_{s}^{b, l}$ ), by the estimate:

$$
\left|\phi^{s}(x)-\Phi_{s}^{b, l}(x)\right| \leq \beta_{l} / 8 \varepsilon_{s} k_{b, s}^{2}\left(x \in R^{1}\right) .
$$

The estimate clearly shows that potentials $\phi^{s}(x)$, $\Phi_{s}^{b, l}(x)$ take arbitrary small magnitude of difference at each of the cases: $\beta_{l}<<1$ (with arbitrary fixed, positive $\left.\varepsilon_{s}, k_{s}, \varepsilon_{b}, k_{b}\right) ; \varepsilon_{s} k_{s}^{2}<<\varepsilon_{b} k_{b}\left(\beta_{l}>0\right.$, fixed). (iii) At extreme magnitude of contour charges, the effect of the modulated surface potential is either identical with that of the leading term $\beta_{l} \phi^{s}$ or negligible, on the phase contour of technological anomalies.

For the comparison on the phase contour $l$ we have to consider the values of $\phi^{s}(0), \Phi_{s}^{b, l}(0)$, using the key relation:

$$
\Phi_{s}^{b, l}(0)=\phi^{s}(0)-\beta_{l}^{2} C_{s}^{b, l} / 16 \pi k_{b, s}^{4}
$$

Here $C_{s}^{b, l} \equiv \int_{0}^{\infty} \frac{\xi^{2} d \xi}{\left[\lambda(\xi)+\varepsilon_{s}\left(k_{s}^{2}+\xi^{2}\right)\right]\left[1+\left(\beta_{l} / 4 k_{b, s}^{2}\right)^{2} \xi^{2}\right]}$.

Relation (3.4) now directly shows that the rest term $\beta_{l}^{2} C_{s}^{b, l} / 16 \pi k_{b, s}^{4}$ tends to zero, for $\beta_{l}$ getting infinitely small, i.e. $\Phi_{s}^{b, l}(0) \approx \phi^{s}(0)$, at $\beta_{l}<<1$. On the other hand, at $\beta_{l} \rightarrow \infty$ it follows that $\beta_{l}^{2} C_{s}^{b, l} / 16 \pi k_{b, s}^{4}$ is tending to $\phi^{s}(0)$. Thus the specific effect appears : $\Phi_{s}^{b, l}(0)$ is vanishing, at $\beta_{l} \gg>1$.

\section{Acknowledgment}

This investigation is devoted to the 125-th anniversary of the Sofia University "St Kliment Ohridski” (1888 - 2013).

\section{References}

[1] A. R. Adams, Sobolev Spaces, Academic Press: New York, 1975.

[2] N.W. Ashcroft, N.D. Mermin, Solid States Physics, Holt, Rinehart and Winston: New York, 1975.

[3] D. Bedeaux, J. Vlieger, Optical Properties of Surfaces, Imperial College Press: London, 2001.

[4] T. Boev, Mathematical models for electrostatics of heterogeneous media (in Electrostatics, edit. by H. Canbolat - Part 4 Mathematical Modelling, 83 - 100), InTech - ISBN 978-953-51-0239-7 (March, 2012); view on line: www.intechopen/books/electrostatics .

[5] Ph. Ebert, Chen Xun, M. Heinrich, M. Simon, K. Urban, M.G. Lagally, "Direct Determination of the Interaction between Vacancies on $\operatorname{InP}(110)$ Surfaces", Phys. Rev. Letters, vol 76, no 12, 18 March. 1996.

[6] J. Gibbs, The Scientific Papers, 1, Dover: New York, 1961.

[7] M. Heinrich, Ph. Ebert, M. Simon, K. Urban, M.G. Lagally, "Temperature Dependent Vacancy Concentrations on InP(110) Surfaces", J. Vac. Sci. Technol. A., vol 13, no 3, May/Jun 1995.

[8] J. Israelishvili, Intermolecular and Surface Forces, Academic Press: London, 1991.

[9] L. Landau, S. Lifschitz, Lectures on Modern Physics, vol. VIII Electrodynamics of Solids, Nauka: Moskow, 1982 (in Russian).

[10] B. Radoev, T. Boev, M. Avramov, "Electrostatics of Heterogeneous Monolayers", Adv. In Colloid and Interface Sci., vol 114-115, pp. 93101,2005 . 